\title{
Recent results and prospects on exploring the helicity structure of the proton at RHIC in high-energy polarized proton-proton collisions
}

\section{Jan Balewski*}

Massachusetts Institute of Technology, Department of Physics, Cambridge, MA 02139

E-mail: balewskiamit.edu

\section{On behalf of the STAR Collaboration}

The STAR Collaboration is pursuing measurements to help understand the origin of the proton spin. The gluon polarization is accesible via measurements of the longitudinal double-spin asymmetry, $A_{L L}$, for inclusive charged and neutral pions and inclusive jet production. A recent global analysis of polarized DIS data and RHIC spin data disfavors extreme gluon polarization scenarios. The measurement of a parity violating single-spin asymmetry, $A_{L}$, for $W^{+}\left(W^{-}\right)$bosons produced in longitudinally polarized $p+p$ collisions at $\sqrt{s}=500 \mathrm{GeV}$, is sensitive to helicity distribution functions for quarks and anti-quarks. The charge-sign reconstruction of charged leptons from $\mathrm{W}$ decays at forward rapidity requires an upgrade of the STAR tracking system based on a novel design of triple-GEM detectors.

European Physical Society Europhysics Conference on High Energy Physics, EPS-HEP 2009,

July 16 - 222009

Krakow, Poland

\footnotetext{
* Speaker.
} 


\section{Gluon helicity measurement}

The gluon helicity distribution function, $\Delta g(x)$, can be probed at RHIC in polarized protonproton collisions by measuring the longitudinal double-spin asymmetry, $A_{L L}$, for a variety of processes involving gluons in the initial state. The sensitivity of a given partonic subprocess depends on the magnitude of the partonic asymmetry, $\hat{a}_{L L}$, which in turn depends on the type of scattered partons involved.

The experimentally measured $A_{L L}$ can be formulated as the ratio of the respective polarized and unpolarized cross-sections. Both cross-sections are calculated employing QCD factorization as a convolution of distribution functions, calculable hard partonic cross-sections at higher orders in $\mathrm{pQCD}$ and fragmentation functions. The data are directly compared to $A_{L L}$ predictions based on PDFs with different gluon polarization scenarios. Inclusive jet data have been included in global analyses with DIS data to constrain $\Delta g(x)$.

\section{Experiment}

The experimental determination of $A_{L L}$ requires three concurrent measurements: a) beam polarization $\left(P_{1(2)}\right)$, b) spin dependent relative luminosity $(R)$ and c) spin dependent event yields $\left(N_{i j}\right)$ of the physics processes of interest

$$
A_{L L}=\frac{\sigma_{++}-\sigma_{+-}}{\sigma_{++}+\sigma_{+-}}=\frac{1}{P_{1} P_{2}} \frac{N_{++}-R N_{+-}}{N_{++}+R N_{+-}}
$$

The STAR experiment is one of the two large collider experiments at the Relativistic Heavy Ion Collider (RHIC). RHIC provides polarized proton beams with a center-of-mass energy of

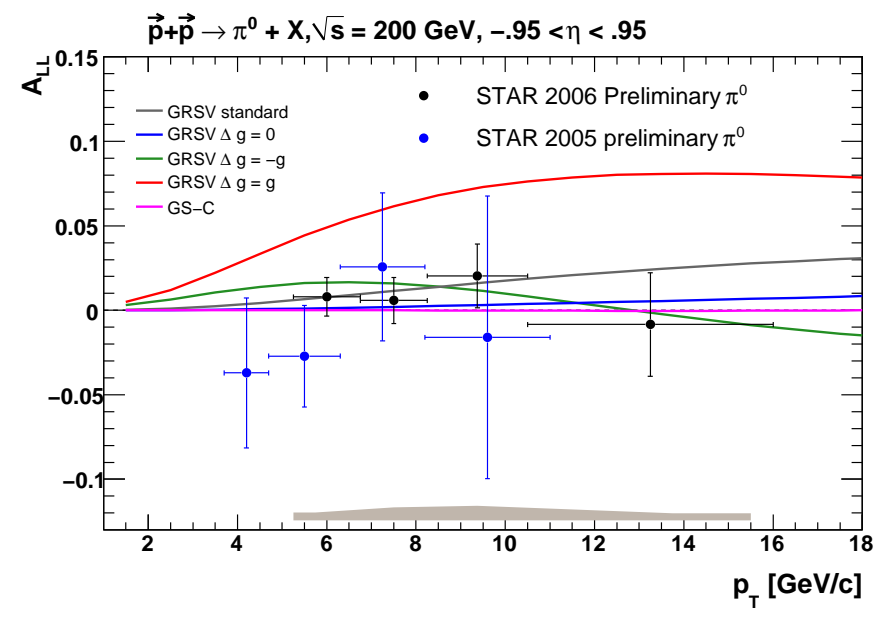

Figure 1: $A_{L L}$ measured at STAR for inclusive $\pi^{0}$ at mid-rapidity at $\sqrt{s}=200 \mathrm{GeV}$. $\sqrt{s}=200-500 \mathrm{GeV}$. Each beam consists of up to 120 bunches, with alternating patterns for the sign of the polarization assigned to the bunches. The direction of polarization axis (transverse or longitudinal) of each beam can be selected using spin rotator magnets installed around the STAR interaction region. The magnitude of the beam polarization was measured and was typically at the level of 50$60 \%$ during the 2006 running period.

The following components of the STAR detector are critical for

gluon polarization measurements. Barrel (BEMC) and endcap (EEMC) electromagnetic calorimeters with full azimuthal coverage span pseudo-rapidities $|\eta| \leq 0.98,1.08 \leq \eta \leq 2.0$, respectively [1]. The forward pion detector (FPD) [2] spans pseudo-rapidity $3.1 \leq \eta \leq 4.4$. The STAR 
Time Projection Chamber (TPC) allows reconstruction of charged tracks with $|\eta|<1$.3. BeamBeam Counters (BBC) cover $3.3 \leq \eta \leq 5.0$ and serve as a minimum-bias trigger and allow the measurement of spin dependent relative luminosities.

\section{Results}

The STAR Collaboration measured unpolarized cross-sections for charged [3] and neutral pions 㺻 produced in $p+p$ collisions at $\sqrt{s}=200 \mathrm{GeV}$. Those measurements agree well with pQCD calculations [5], 6], which provides an important baseline for the interpretation of neutral and charged pion $A_{L L}$ in the context of pQCD calculations.
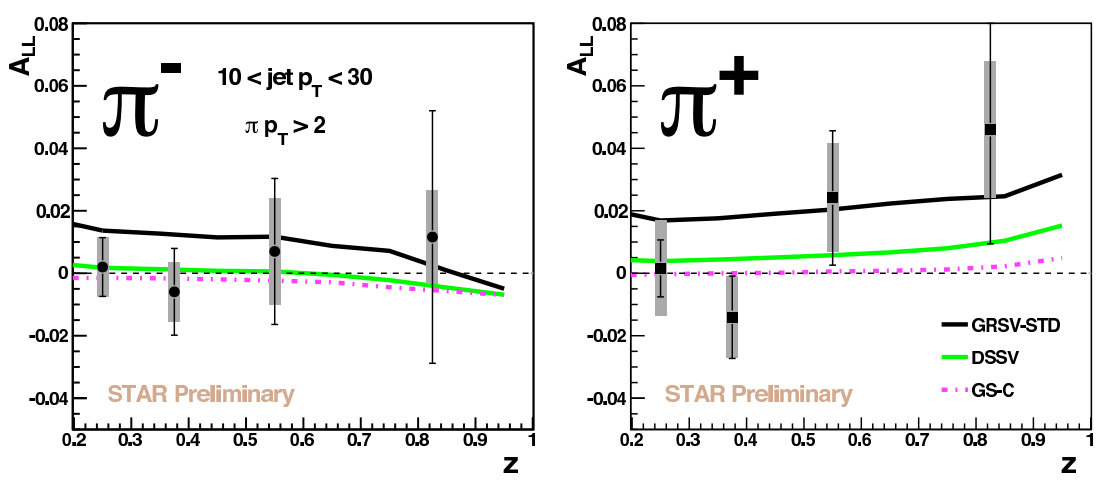

Figure 2: $A_{L L}$ measured at STAR for inclusive charged pions at mid-rapidity at $\sqrt{s}=200 \mathrm{GeV}$.
$A_{L L}$ for $\pi^{0}$ measured at mid-rapidity with the BEMC in the $p_{T}$ range 5$16 \mathrm{GeV} / \mathrm{c}$ is shown in Figure 1. It rules out extreme gluon polarization scenarios in the context of the GRSV-MAX parameterization [T]. $A_{L L}$ for $\pi^{0}$ at forwardrapidity was measured with the EEMC

and FPD [8]. The predicted magnitude of $A_{L L}$ at forward rapidity is significantly smaller in comparison to mid rapidity. Current experimental uncertainties in these forward regions are still too large to draw any further conclusion on the gluon polariation.

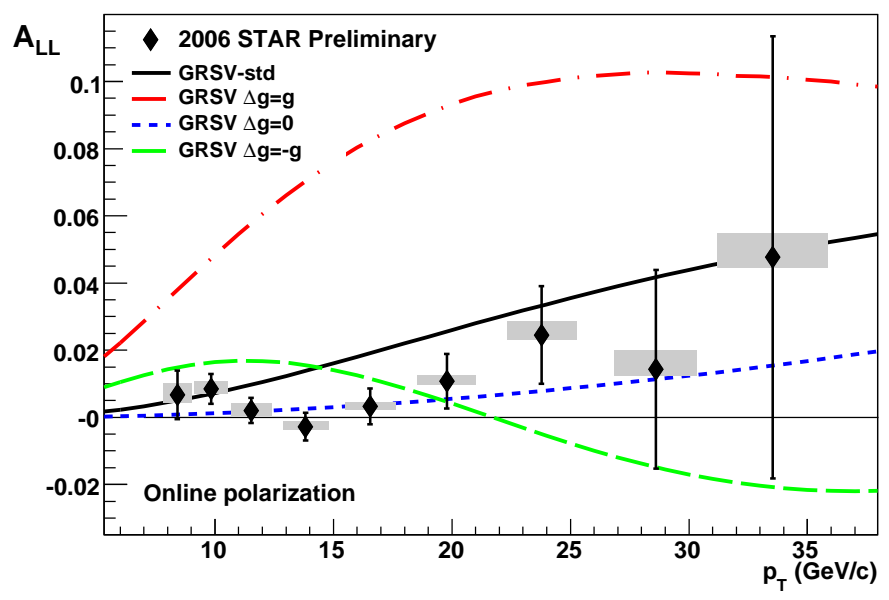

Figure 3: $A_{L L}$ measured at STAR for inclusive jets as a function of $p_{T}$.
High $p_{T}$ charged pions were measured in the STAR TPC. Excellent dE/dx calibration of the TPC [9] allowed discrimination against other charged hadrons for momenta up to $15 \mathrm{GeV} / \mathrm{c}$. Since the STAR trigger is dominated by the response of an electromagnetic calorimeter, the recorded events are biased toward triggered jets containing a leading neutral $\pi^{0}$. In the off-line analysis it was required that the charged pion is part of the away-side jet. 
The majority of recorded events are di-jets from the hadronization of a $2 \rightarrow 2$ partonic scattering processes. Therefore, the $p_{T}$ of the reconstructed triggered jet was used as a measure of the partonic $p_{T}$ and the extracted $A_{L L}$ [10] was determined as a function of $z$ defined as $z \equiv p_{T}($ charged $\pi) / p_{T}($ trigger jet $)$.

Comparison of the measured $A_{L L}(z)$ [10] to pQCD predictions [11] is shown in Figure 2. Future measurements of charged pion production with higher statistical precision would allow additional constraints on the gluon polarization through a global analysis.

STAR has published the differential inclusive jet cross section and spin asymmetry $A_{L L}$,12, [13]. In 2006 more precise $A_{L L}$ data were obtained with $p_{T}$ up to $40 \mathrm{GeV} / \mathrm{c}$ and $\eta \in[-0.7,0.9]$, as shown in Figure 3. Jets were reconstructed using a STAR implementation of the mid-point cone algorithm [14] with cone radius of 0.7 radians, a seed energy of $E_{T}>0.5 \mathrm{GeV}$, association threshold $E_{T}>0.1 \mathrm{GeV}$, and split fraction of 0.5. The measured $A_{L L}$ for inclusive jets is shown in Figure 3 . STAR inclusive jet $A_{L L}$ has been already incorporated in a global analysis [15] and provides a strong constraint on $\Delta g(x)$ for $0.05<\mathrm{x}<0.2$.

\section{Prospects for measurement of the sea quark polarization}

Polarized DIS [16] experiments show that the total quark and anti-quark contribution to the proton spin, summed over all flavors, is surprisingly small. Inclusive DIS measurements provide sensitivity only to the combined contributions of quarks and anti-quarks. However progress in constraining $(\Delta \bar{u}(x)-\Delta \bar{d}(x))$ has been achieved by incorporating SIDIS data [17, 18] in global QCD analysis [19]. The production of $\mathrm{W}$ bosons in high energy collisions of polarized protons at RHIC provides independent and direct sensitivity to the helicity of $\mathrm{u}, \bar{u}, \mathrm{~d}$, and $\bar{d}$ quarks in the proton through the maximal parity violating single spin asymmetry $A_{L}$.

The rapidity of produced $\mathrm{W}$ bosons carries information about the momentum fraction of the initial $q \bar{q}$ system $x_{1(2)}=\frac{M_{W}}{\sqrt{s}} e^{+(-) y_{W}}$. At STAR only the final state charged lepton is measured in $\mathrm{W}$ decays. The quark, anti-quark momentum fractions $x_{1}, x_{2}$ can be related in an approximate way to the measured lepton $p_{T}$ and rapidity if lepton rapidity $\left|y_{l e p}^{l a b}\right|>1$. The weak production processes involved are parity violating, yielding large longitudinal single spin asymmetries in regions dominated by quarks. At STAR we will measure the relative difference between the yield of left-handed $\left(N_{-}^{W}\right)$ and right-handed $\left(N_{+}^{W}\right)$ Ws produced in collision of a longitudinally polarized left-handed and right-handed proton beam with an "unpolarized" proton beam, i.e. for this measurement polarization of one of the beams will be avearged to zero. The theoretical framework of the measurement of the longitudinal single-spin asymmetry $A_{L}$ for Ws as a function of the leptonic rapidity is well developed and has been presented in [20]. The global analysis framework has recently been completed to allow the extraction of quark and anti-quark distribution functions using measured $A_{L}$ at RHIC as input in a full global analysis [19]. Based on resummation calculations [20] and assuming STAR will acquire integrated luminosity of $300 \mathrm{pb}^{-1}$ with an average polarization of $70 \%$ we expect to confirm the known polarization of quarks and deepen our understanding of anti-quark polarization, in particular for the $\bar{u}$. 


\section{Conclusions}

The recent STAR inclusive jet results along with the PHENIX neutral pion results have been used for the first time to constrain $\Delta g(x)$ in a NLO global analysis (DSSV) along with semiinclusive and inclusive DIS data [15]. The RHIC data sets provide strong constraints on $\Delta g(x)$ for $0.05<x<0.2$. Inclusive measurements, such as inclusive jet production, integrate over a fairly large $x$ region for a given jet transverse momentum region. While those measurements provide a strong constraint on the value of $\Delta g(x)$ integrated over a range in $x$, those measurements do not permit a direct sensitivity to the actual $x$ dependence. This motivates the need for correlation measurements in polarized proton-proton collisions, such as di-jet measurements. Di-jet production will play a critical role in the future, deepening our understanding of $\Delta g(x)$, in particular, by constraining its shape. The analysis of STAR dijet data from Run 5 and Run 6 is currently underway.

\section{References}

[1] K.H. Ackermann et al. [STAR Collaboration], Nucl. Instrum. Meth. A 499, 624 (2003); M. Beddo et al., ibid. 725; C.E. Allgoweret al., ibid. 740

[2] J. Adams et al., Phys. Rev. Lett. 92 (2004) 171801

[3] J. Adams et al., Phys. Lett. B 637 (2006) 161, nucl-ex/0601033; Y.Xu for the STAR Collaboration, Eur. Phys. J. C (2009) 62:187-190, arXiv:0901.0692

[4] M.J. Russcher et al, 2007 J. Phys. G: Nucl. Part. Phys. 34 S1033-S1036

[5] B. Jäger et al, Phys. Rev. D 67, 054005 (2003)

[6] B.A. Kniehl et al, Nucl. Phys. B597, 337 (2001)

[7] M. Glück et al., Phys.Rev. D63 (2001) 094005

[8] S.W. Wissink et al. for the STAR Collaboration, in Proceedings of the 18th International Symposium on Spin Physics (AIP, to be published)

[9] Y. Xu et al., arXiv:0807.4303v1

[10] A.Kocoloski for the STAR Collaboration, arXiv:0905.1033

[11] D. Florian et al., Phys.Rev.Lett. 79 (2009) 114014, http://arxiv.org/abs/0904.4402

[12] B.I. Abelev et al., Phys.Rev.Lett. 97 (2006) 252001, hep-ex/0608030

[13] B.I. Abelev et al., Phys.Rev.Lett. 100 (2008) 232003, arXiv:0710.2048 [hep-ex]

[14] G. C. Blazey et al., arXiv:hep-ex/0005012.

[15] D. Florian et al. Phys.Rev.Lett. 101072001 (2008), arXiv:0904.3821

[16] B.W. Filippone and X.D. Ji, Adv. Nucl. Phys. 26, 1 (2001)

[17] A. Airapetian et al., [HERMES Collaboration], Phys. Rev. D 71, 012003 (2005)

[18] M. Alekseev et al., [COMPASS Colaboration], Phys.Lett. B 660, 458 (2008)

[19] D. de Florian et al., Phys.Rev.Lett. 80 (2009) 034030, arXiv:0904.3821v1 [hep-ph]

[20] P. M. Nadolsky and C. P. Yuan, Nucl. Phys. B666, 31 (2003), hep-ph/0304002 